
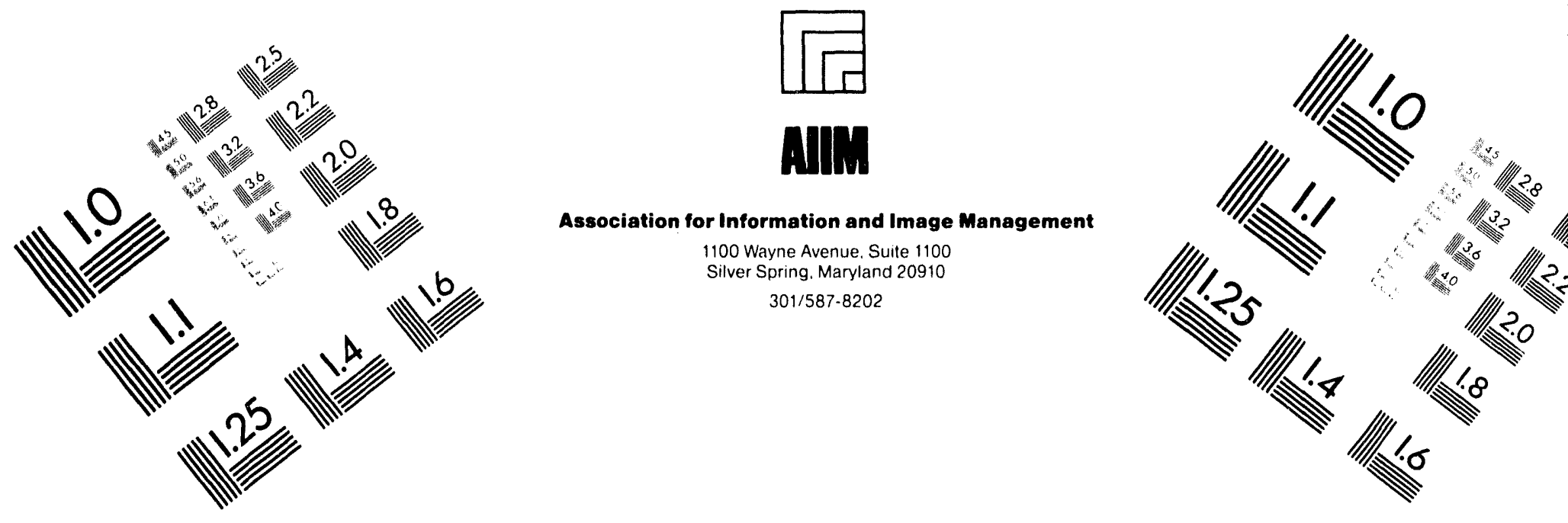

\title{
Centimeter
}

${ }_{1}$ Inches
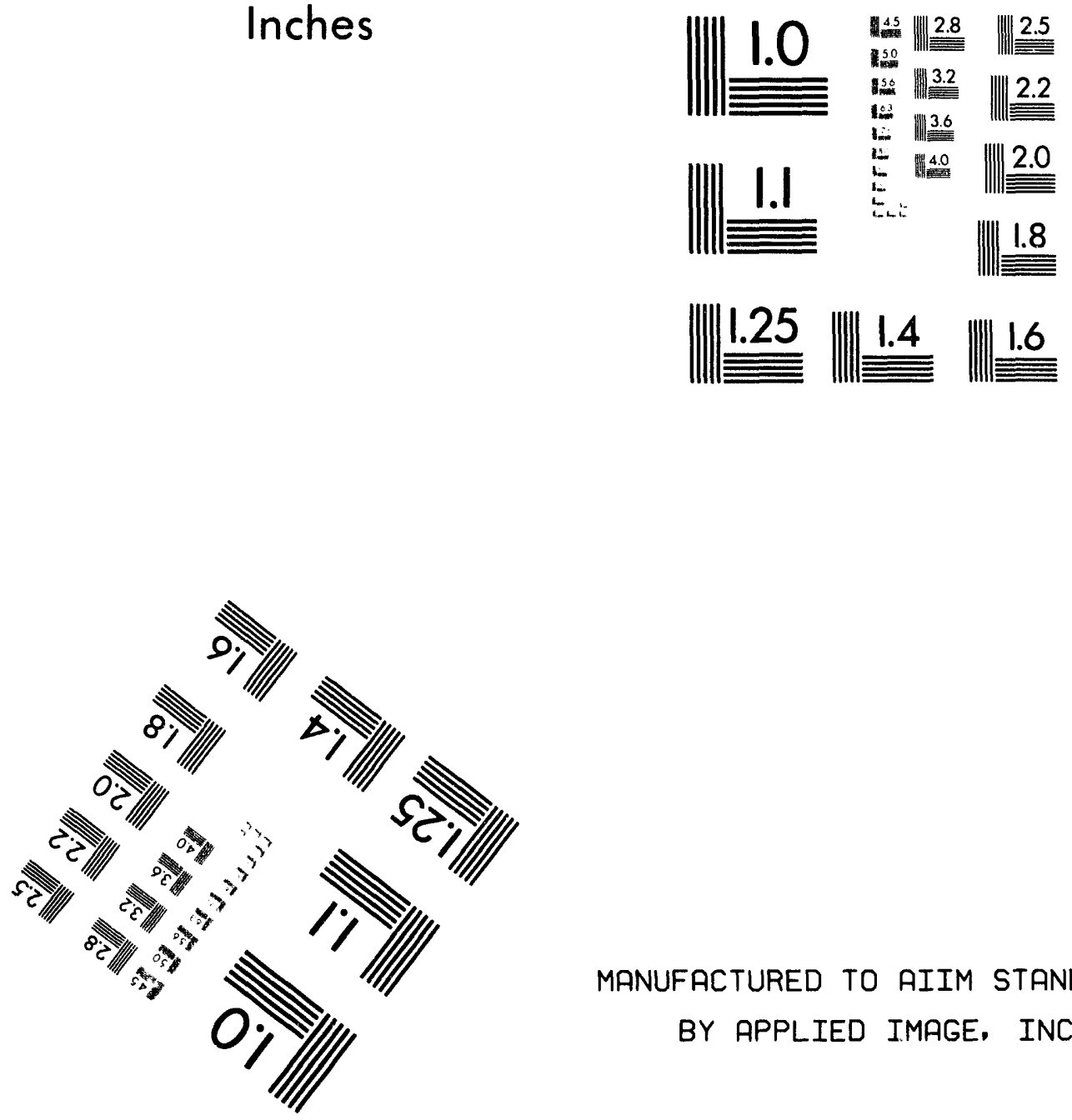

MANUFACTURED TO AIIM STANDARDS

BY APPLIED IMAGE, INC.

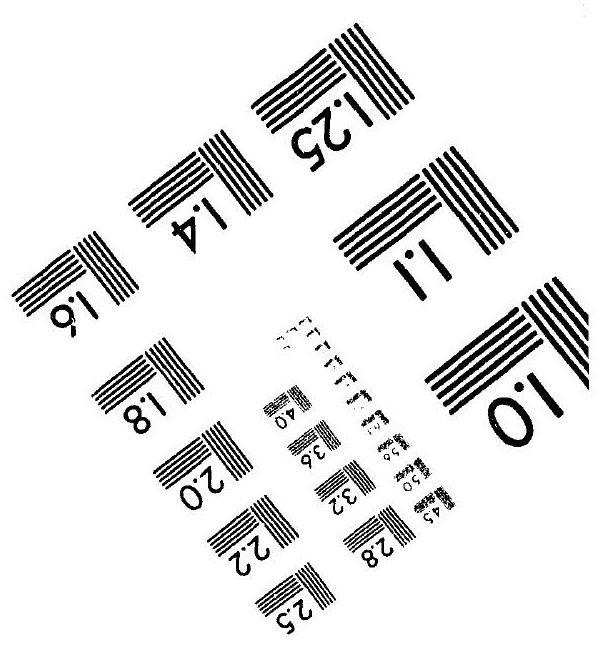



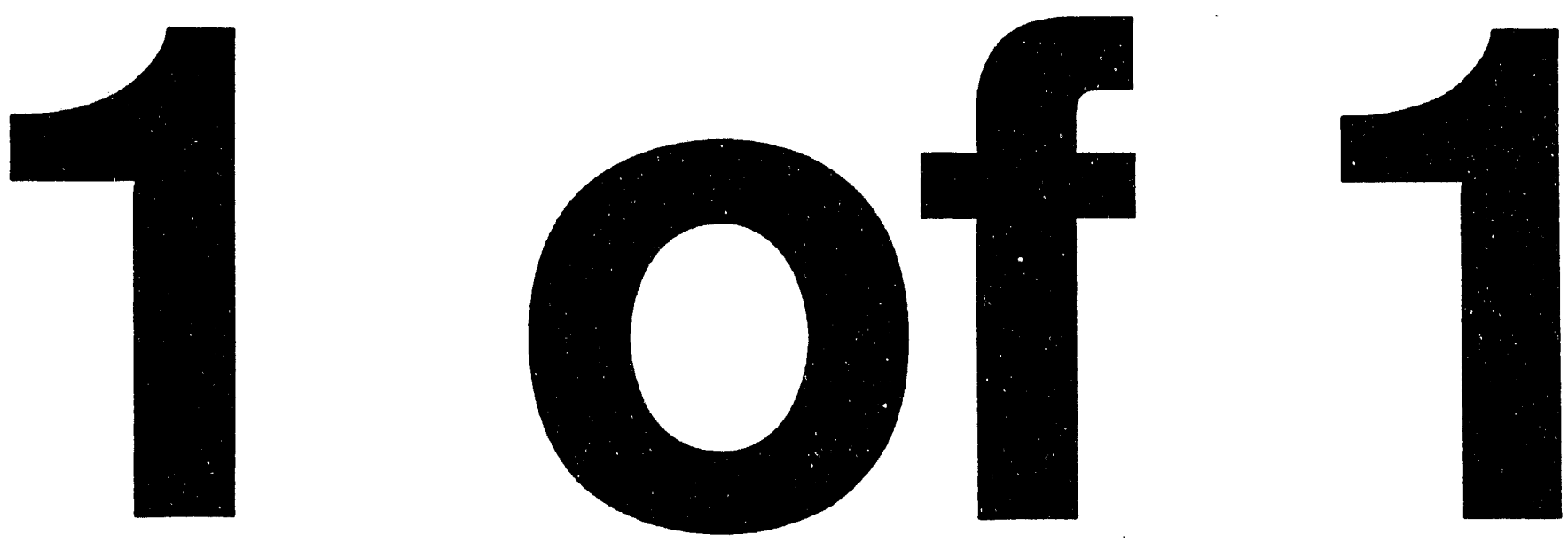
PNL-SA-23362

POWER SYSTEM APPLICATIONS FOR PASC CONVERTER SYSTEMS

M. K. Donnelly

R. M. Johnson ${ }^{(2)}$

April 1994

Presented at the

1994 Institute of Electrical and Electronics

Engineers/PES T\&D Conference

April 10-15, 1994

Chicago, Illinois

Prepared for

the U.S. Department of Energy

under Contract DE-AC06-76RLO 1830

Pacific Northwest Laboratory

Richland, Washington 99352

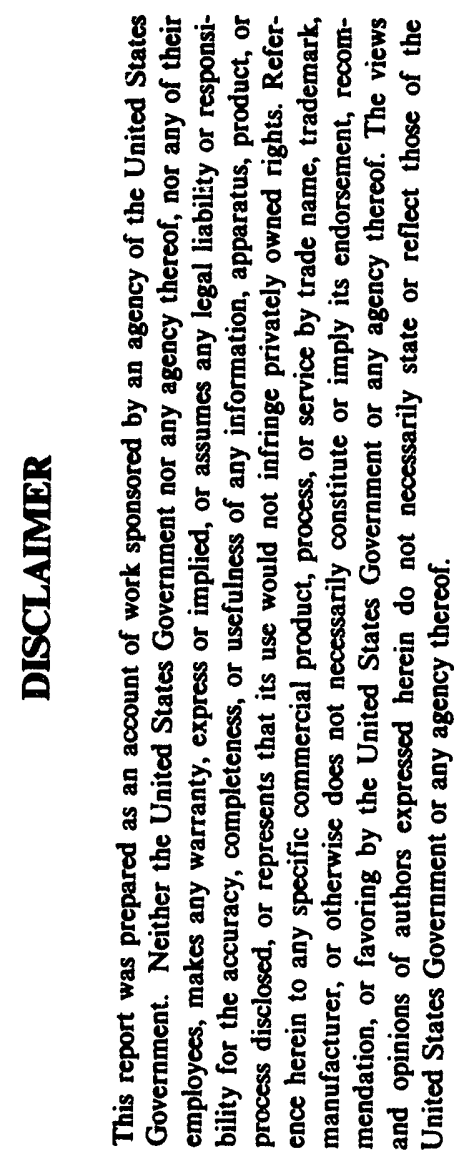

(a) Montana State University

Bozeman, Montana 


\section{Power System Applications for PASC Converter Systems}

\author{
M. K. Donnelly, Member, IEEE \\ Pacific Northwest Laboratory \\ Richland, Washington
}

\author{
R. M. Johnson, Member, IEEE \\ Montana State University \\ Bozeman, Montana
}

\begin{abstract}
This paper shows, using computer EMTP simulations, some preliminary results of applying pulse amplitude synthesis and control (PASC) technology to single-source level voltage converter systems. The method can be applied to any single terminal pair source with appropriate modifications in power extraction interface and computer control program to match source and load impedance characteristics. The PASC realization as discussed here employs banks of transformers, one bank per phase, in which the primaries are connected in parallel through a switch matrix to the dc source. Two opposite polarity primaries per transformer are pulsed alternatively in time to produce an oscillatory sinusoidal output waveform.

PASC conversion system capabilities to produce both leading and lagging power factor power output in singlephase and three-phase $\Delta$ or $Y$ configurations are illustrated. EMTP simulations are used to demonstrate the converter capabilities. Also included are discussions regarding harmonics and potential control strategies to adapt the converter to an application or to minimize harmonics.
\end{abstract}

Keywords: static inverter, dc power conversion, steppedwave inverter

\section{INTRODUCTION}

Recently, pulse amplitude synthesis and control (PASC) multiple-source and single-source converter systems have been described in the literature $[1,2]$. The PASC technique uses digital computer-controlled power waveform synthesis techniques to produce a desired output from either single or multiple dc sources. The type of PASC converter presented here is made up of several (to hundreds of) three-winding transformers. Each transformer consists of two identical primaries and a single secondary.
Individually, each transformer makes up one $\mathrm{n}^{\text {th }}$ of the entire MVA rating of the converter, where $n$ is the number of transformers comprising the system. This topology allows the switching components of a PASC converter to be rated at the low power specifications of a single PASC transformer rather than the high power rating of the entire converter. A single-phase PASC converter is shown schematically in Fig. 1. The extension to three-phase is straightforward, with the composite secondary winding becoming one leg of the threephase converter. The three-phase secondaries can be connected in either $\Delta$ or $Y$.

Two advantages of the PASC technology can be readily seen from Fig. 1. The first is the ability to incorporate lowpower solid-state devices as switches in the PASC converter. This aspect of the PASC converter is analogous to the parallel strings of thyristors commonly used in conventional converters to reduce the current in any single valve string of the converter. A problem with this method of reducing current in individual devices lies in the difficulty in obtaining an equal current split between parallel strings. For the PASC converter, however, the electrical isolation provided by the consolidation transformers alleviates these problems.

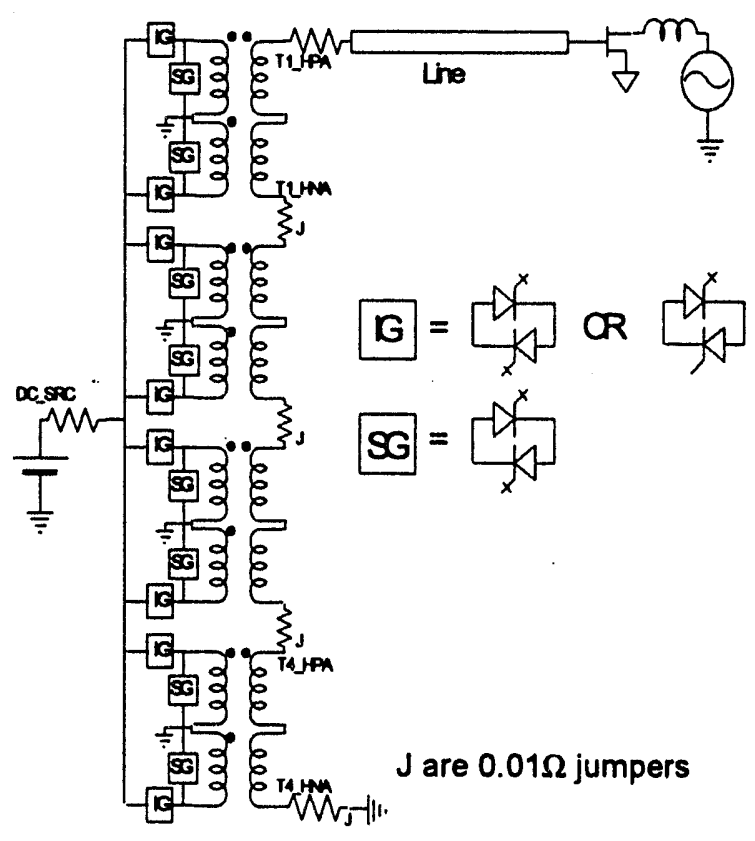

Fig. 1. 4-Core PASC Converter 
A second advantage of PASC technology as seen from Fig. 1 is the aforementioned electrical isolation between source legs of the primaries. While isolation and consolidation are not concerns when dealing with a single dc source, they become crucial concerns in the case of new generation and storage technologies with distributed sources such as maguetohydrodynamics (MHD), wind and solar, batteries, and asynchronous ac generation.

While $[1,2]$ have described some of the benefits derived from PASC converter technology from the point of view of magnetohydrodynamic energy conversion, this paper examines the converter as it applies to issues in the generationtransmission interface. Previously published literature has treated PASC as an ac drive. The results contained in this paper present PASC as a three-phase converter operating into a small network as shown in Fig. 2. In this configuration, issues such as harmonics, real and reactive power control, and converter control algorithms can be addressed. System dynamic performances issues are not addressed in this paper. However, simulations and experimentation on a prototype converter have shown that both real and reactive power modulation are straightforward to implement.

Although many different PASC converter configurations can be realized, the configuration chosen for discussion here is the single-phase voltage source converter shown in Fig. 1. In this configuration, the primaries of four power transformers are connected in parallel across a dc source while the secondaries of the transformers are connected in series across the load. Three-phase converters are constructed of three single-phase converters interconnected as appropriate to the application. Both series power-injection gates and shunt shorting gates are required for each transformer for reasons described previously [2]. Although very low harmonic content sinusoidal output waveforms can be generated by going to higher-level PASC converters, a four-level converter has been chosen for consideration here because its gate count is comparable to standard 12-pulse converters, and preliminary work has shown that a controlled PASC voltage converter can produce significantly fewer harmonic filtering requirements for many applications.

The remainder of this paper is presented as follows: Section II provides a detailed description of the converter configuration used for simulation; Section III provides some discussion of the simulation methods. In Section IV, some applications for the converter are proposed; Section $V$ presents the pertinent simulation results, and Section VI provides conclusions.

\section{DESCRIPTION}

A complete single-phase, four-transformer, single-source PASC converter is shown in Fig. 1. Fig. 1 also depicts the A phase of the simulation configuration used in the EMTP studies. A $Y$ secondary is constructed as shown in the diagram. In a $\Delta$ configuration, node T4_HNA is not connected

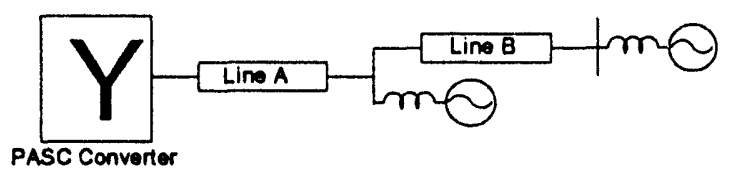

Fig. 2. Study System

to ground, but is connected to the dotted winding of the B phase.

In its most general configuration, a PASC converter is capable of bidirectional power flow at any power factor, leading or lagging. Harmonics are kept to a minimum using computer-controlled switching. Because most of the gates enjoy soft switching at low frequencies, switching losses are also relatively low. To provide a smooth transition from inverter to ectifier and back, the injection gates labeled IG in Fig. 1 would need to be antiparallel gate turn-off thyristors (GTOs) or MOS-controlled thyristors (MCTs). In many applications, MCTs will likely replace GTOs [3]. Inverteronly operation would require a GTO in the forward direction in antiparallel with a standard thyristor for the injection gate. Finally, when used as an ac drive, a forward GTO alone is adequate. It is desirable to use the minimal configuration for a given application because the valve facing the dc voltage source is subjected to relatively high reverse blocking voltages $(2 \mathrm{~V} \mathrm{dc})$.

The shorting gates, labeled SG in Fig. 1, are shown as antiparallel GTOs. As the name implies, the function of the shorting gate is to create a physical short-circuit across the primary of a "nonactive" transformer. This action effectively removes the transformer from the circuit by creating a short across the secondary during the time the control algorithm is not calling for power from that particular source (e.g., near the voltage zero crossing, all transformers are "shorted"). Each transformer must have the ability to be shorted in either direction. This can be accomplished either by antiparallel GTOs in one primary or by a single opposing GTO in each primary. Fig. 1 shows the safest configuration of antiparallel GTOs in each primary for redundancy.

As stated previously, the injection gates must be capable of blocking twice the applied dc voltage. This voltage is applied, for example, to the "negative polarity" injection gates while the positive polarity injection gates on the same transformer core are conducting. Conversely, the shorting gates must withstand unity times the applied dc voltage when either of the injection gates are conducting.

Continuity of source loading is maintained by connecting each source to corresponding transformers on all three phases.

\section{SIMULATION AND MODELING}

A working 24-kVA prototype converter is currently being evaluated at Montana State University $[4,5]$. The transform- 
ers from the prototype were tested and faithfully modeled as three-winding, saturable transformers in an EMTP. The transformers used in the prototype are standard resinencapsulated, dry-type, general purpose, Type QB transformers. Each of the eight transformers used in the prototype is rated 3-kVA, 4-winding, 120/240 to 120/240. The primary windings are isolated in the PASC implementation, and the secondaries on each transformer are connected in series to form a 2:1 step-up as viewed from any single primary.

A rudimentary power system was modeled and connected to the EMTP model of the PASC converter as shown in Fig. 2. Transmission line $A$ is 90 miles long and is modeled with pi sections. Transmission line $B$ is 120 miles long and is also modeled using pi sections. The two source-reactance pairs are first-order synchronous machines.

Using this configuration, tests were conducted to examine the characteristics of a PASC converter with respect to harmonics, real and reactive power controllability, and control algorithm effectiveness.

PSpice simulations were concurrently conducted to validate the EMTP results, to provide more detailed analysis of switching transients, and to aid in the design process.

The $\mathrm{T}$ transformer model used in the PSpice simulations is shown in Fig. 3. The ideal transformer has a step-up secondary-to-primary ratio of a. If the magnetizing, hysteresis, and core currents are of small per unit value, the shunt Rc/Lm portion of the circuit can be neglected. However, in pulse applications, particularly at high frequencies of operation, the core and hysteresis losses can be high so in the model developed here the shunt portion of the model is retained. In the PSpice model used here, the source injection (Rs) and shorting gates (SNp or SNs) are implemented as control signal-initiated, time-variable resistors, with the on and off resistances adjusted to incorporate the effects of turns ratio and corresponding element values.

For the two-terminal case, only a single winding is used for the primary because it is easier in PSpice to simply switch the polarity of the input source rather than incorporate another primary winding circuit like those shown in Fig. 3.

Following this general procedure, the PSpice simulation will contain as many transformers in parallel as the number of unipolar steps to be used in approximating the desired output waveform.

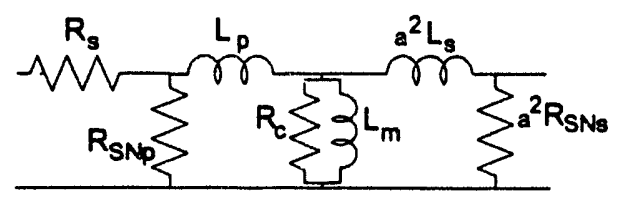

Fig. 3. Transformer " $T$ " Model for PSpice

\section{APPLICATIONS}

The PASC converter concept was developed for the U.S. Department of Energy as a means to consolidate the electrical output of an MHD generator in space [5]. Electrical isolation between electrode pairs on the MHD generator and control flexibility were emphasized. These two features are also important in many modern power system applications. This section summarizes key features of a PASC converter in some potentially attractive applications.

\section{A. Multisource Applications}

Many contemporary generation and storage technologies consist of numerous separate sources close to one another so as to make up a moderate to large conglomerate facility. Included in this category are batteries, solar arrays, wind farms, fuel cells, and MHD. Future applications in which electrical isolation of the separate sources may prove useful include asynchronous generation at hydroelectric plants. A common factor in all of these generation and storage technologies is that electrical energy at several different voltages, and possibly frequencies, must be consolidated at some point.

Each transformer of a PASC converter can be controlled in a manner so as to optimally load the corresponding source with little or no effect on other sources in the facility. Removing a source from service is accomplished by opening the corresponding injection gates and closing the shorting gates for the source. For multisource applications, PASC may allow control of individual sources for optimal efficiency.

\section{B. Control Methods}

Several loops can be used to control the output parameters of a PASC converter. At the most basic level, a simple feedback loop is designed to control the amount of real power delivered or consumed by the converter. The converter is also capable of providing reactive support. Again, a simple feedback loop can be designed to provide proper tracking of a reactive power set point. Real and reactive power modulation can be achieved using more sophisticated controllers.

Much of the initial research involving PASC control algorithms has been conducted in the area of harmonic minimization. A fundamental principle of classical control theory was evaluated for its usefulness in minimizing harmonics in a PASC converter.

The internal model principle of control theory states that a "structurally stable" design always incorporates a model of the reference to be input in the forward loop and/or the disturbance to be rejected in the feedback path. Francis and Wonham [6] give a general proof of this principle and Franklin and Powell [7] apply the principle to several different signals including a sinusoidal reference. The resulting con- 


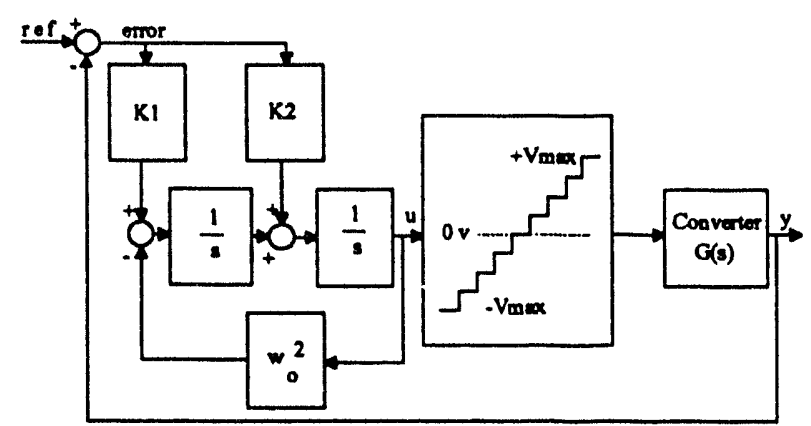

Fig. 4. Controller Model for Sinusoidal Tracking

troller incorporating an oscillator in the forward path is shown in Fig. 4.

The control signal, $u$, is quantized to a number of levels corresponding to the number of levels in the PASC converter. Fig. 4 shows a quantization block for a four-level converter. A control signal to the converter of one-quarter $V \max$ (the first level of quantization) would initiate a gate firing pulse for one of the injection gates shown in Fig. 1.

Research has shown that the gain of the controller in Fig. 4 exerts great influence over the chăracteristics of the harmonics generated by the converter. At low gains, the converter output waveforms resemble those of an idealized classical bridge converter. In fact, in the open loop the converter emulates a stepped-wave inverter with unequally spaced steps of equal magnitude. The step widths are optimized to track a sinusoid. In this mode, switching frequencies are very low and characteristic harmonics can be expected at frequencies corresponding to those of a bridge converter with similar numbers of pulses. As the gain on the controller is increased, the PASC converter begins to more closely resemble a pulse-width modulation (PWM) converter. The harmonics are shifted upward in frequency, and the switching frequency increases. Generally, more loss can be expected at the higher switching frequencies, so a tradeoff exists between losses and low-frequency harmonics.

This phenomenon suggests that a gain scheduling algorithm may be useful in balancing the harmunics and losses from a PASC converter. One scenario that has been investigated involves forming a ratio of low-order harmonics versus switching frequency and adjusting the gain to optimize the ratio. Further research is being conducted in this area.

Some work has also been done to develop algorithms for controlling power from individual sources in the multisource case. To date, this work has been focused primarily on current shuffle circuits for MHD power generation, but the results could certainly be applied to other generation and storage technologies as well.

\section{SIMULATION RESULTS}

A PSpice simulated open-loop response for a four-level sinusoidal input modulation PASC converter was performed to validate similar simulations in an EMTP. The PSpice results showed good correlation with the EMTP results, previous investigations using an in-house FORTRAN simulator, and digitized oscilloscope files taken from the working 24-kVA prototype. A great deal of confidence has been developed for the usefulness of the EMTP model in generating realistic results. Not only have three separate simulation procedures been refined to produce similar results, but virtually every case in which the working prototype has been used to validate modeling has proven successful.

The interaction between a PASC converter and the network can be visualized in almost the same manner as classical first-order synchronous machine dynamics. The computer control algorithm generates a reference sinusoid that the converter will attempt to track. One can speak of a power angle $(\delta)$ between the reference sinusoid and the voltage angle of a distant bus. As the power angle increases to 90 degrees, the real power transfer increases to a maximum. Should the power angle go negative, the converter becomes a rectifier. The reference sinusoid also contains amplitude information. Changes in reference sinusoid amplitude correspond to changes in reactive power delivered, provided that the converter/source configuration can supply the demand. Small changes in reactive power can be accomplished by simply changing the amplitude of the reference. Larger reactive power adjustments may need to involve tap changer activity, variations in source voltage, or additional converter levels.

The simulation results shown in this section were performed using an open-loop control wherein a reference sinusoid was generated as described above and held constant throughout the simulation. This control mode generated a gate firing sequence for a four-level stepped-wave inverter with unequal spacing between steps. The Fourier series representation of the ideal waveform is

$$
f_{s}(t)=\sum_{\substack{n=1 \\ n \text { odd }}}^{\infty} b_{n} \sin \left(n \omega_{0} t\right)
$$

where

$$
\begin{aligned}
b_{n}=\frac{1}{\pi n}\left[\cos \left(n \arccos \left(-\frac{1}{8}\right)\right)+\cos \left(n \arccos \left(-\frac{3}{8}\right)\right)\right. \\
\left.+\cos \left(n \arccos \left(-\frac{5}{8}\right)\right)+\cos \left(n \arccos \left(-\frac{7}{8}\right)\right)\right] .
\end{aligned}
$$

It is interesting to note that the Fourier coefficients of this series are made up of points on Chebyshev polynomials. The THD as calculated from the truncated Fourier series of this idealized representation is $8.35 \%$. Similar analysis on a six-level PASC converter operating in this manner yieided a $5.28 \%$ THD.

Four cases were developed to demonstrate the analogy between synchronous machine operation and PASC converter control. In two cases, $\delta$ was changed to illustrate converter 
operation as an inverter and rectifier. In these studies, the dc source voltage was fixed at $125 \mathrm{~V}$. Another case illustrates reactive support wherein the power angle was held constant, but the dc source voltage was increased by approximately $16 \%$. In a practical application, the reactive support could be provided without changing the dc source voltage. One in several ways would be to change the transformer turns ratio. Small changes in reactive power output can be accomplished by simply changing the reference sinusoid amplitude without changing apparent source voltage amplitude. This change effectively shifts the gate-firing sequence to provide some reactive support. Table I shows some pertinent results from EMTP simulations made on the system shown in Fig. 1.

Table I. Power and THD for Simulation Results

\begin{tabular}{|c|c|c|c|c|}
\hline$\delta($ deg), Vdc & $P(W)$ & $\Theta(V-I)$ & THDi(\%) & THDV $(\%)$ \\
\hline $15,125 V$ & 1,427 & 17.2 & 1.4 & 7.6 \\
\hline $30,125 V$ & 2.783 & 18.8 & 0.7 & 7.6 \\
\hline $15,145 V$ & 1,684 & 43.6 & 1.2 & 7.6 \\
\hline$-7.5,125 V$ & -751 & -206.6 & 2.6 & 7.5 \\
\hline
\end{tabular}

The power factor angle shown in Table I was defined as the angle of the voltage waveform minus the angle of the current waveform. A positive value means that current lags voltage. The THD shown for the voltage waveform was consistently $7.6 \%$ for the four-level converter operating in the open loop. This is close to the predicted value of $8.35 \%$ derived above. Spectral leakage associated with the FFT of the voltage waveform probably accounts for a portion of the difference because the predicted values were derived directly from the Fourier coefficients.

Fig. 5 through 8 show typical waveforms from PASC EMTP simulations on the system of Fig. 1. Fig. 5 shows the line current waveform for the 15-degree power angle, 125-V source case. Fig. 6 shows the spectrum of Fig. 5. Fig. 7 and 8 show the voltage waveform and spectrum for the same case.

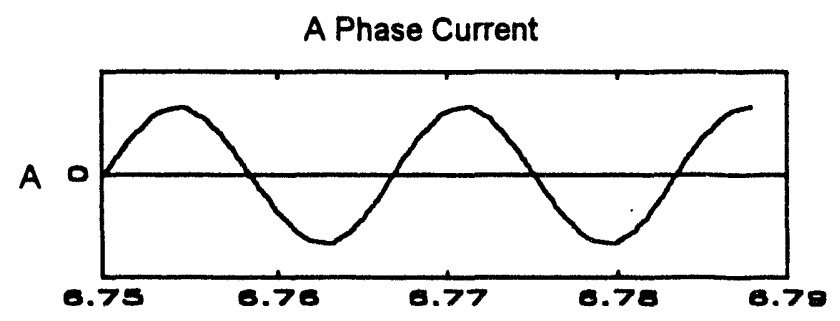

Time (s)

Fig. 5. A-Phase Current for Case 1

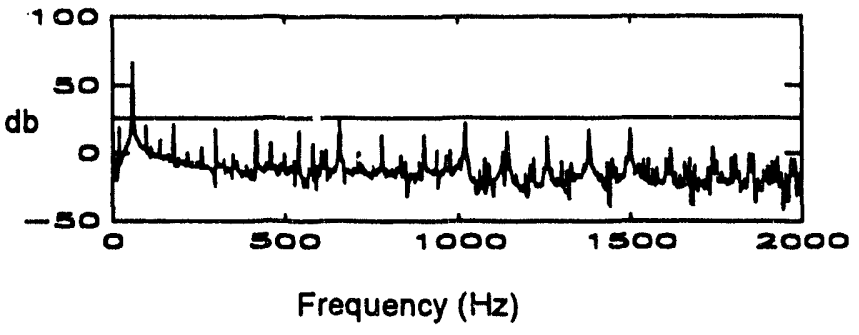

Fig. 6. Spectrum of A-Phase Current

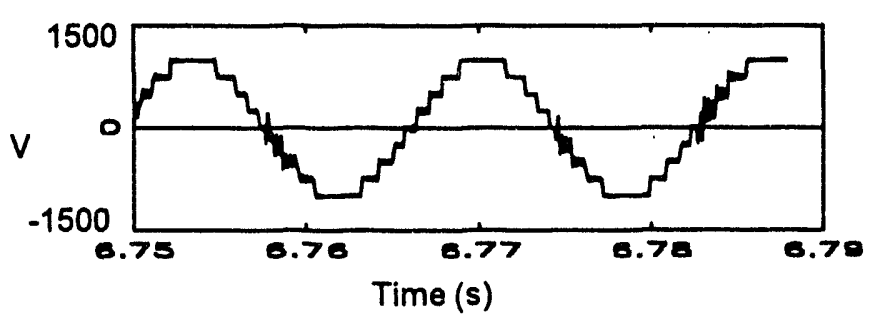

Fig. 7. A-Phase Voltage

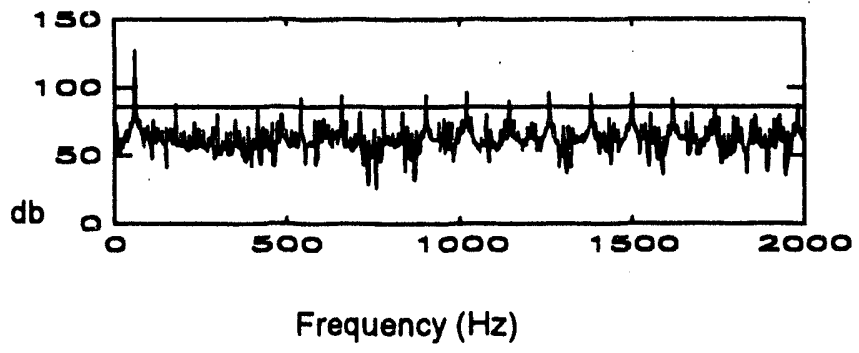

Fig. 8. Spectrum of A-Phase Voltage

The horizontal lines in the FFT plots are $40 \mathrm{db}$ down from the magnitude of the fundamental. The characteristic appearance of the voltage waveform in Fig. 7 begins to look much more like a PWM wavefonn as PASC controller gain is increased.

\section{CONCLUSIONS}

PASC converter technology has been proposed for MHD power consolidation and control applications because of its ability to provide electrical isolation to diverse sources and to provide flexibility of control to both the source and load $[1,2,4,5]$. These properties are desirable not only in MHD 
applications but in a number of other power system applications as well. However, previous research in this area has dealt only with converter control as it relates to MHD electrode configuration and only operating as an ac drive.

This paper has presented simulation results for a PASC converter operating as both an inverter and rectifier into a small electrical network composed of transmission lines and first-order synchronous machines. A potential algorithm for harmonic minimization was presented, but even open-loop results show reasonable harmonic content--on the order of $1 \%$ in the current waveform. The converter was shown to allow real and reactive power control.

In one control scenario discussed above, a reference sinusoid was generated and a simple controller was developed that would closely track the reference. Using this strategy as a building block, more controls could be added to modulate real and reactive power, provide voltage support, optimize source efficiencies, integrate diverse generation and storage technologies, and minimize harmonics. Some potential applications of PASC converter technology were also discussed.

Finally, it was noted that control algorithms can be chosen to make the PASC converter appear to operate similarly to a stepped-wave converter or on the other end of the spectrum as a PWM converter. Higher switching speeds may induce higher losses, and lower switching speeds produce output waveforms with more low-order harmonics.

\section{ACKNOWLEDGEMENT}

Pacific Northwest Labortatory is operated for the U.S. Department of Energy by Battelle Memorial Institute under Contract DE-ACO6-76RLO 1830.

\section{REFERENCES}

[1] R. M. Johnson, M. K. Donnelly, and K. Marcotte, "Pulse-Amplitude-Synthesis-and-Control (PASC) Consolidation/Inversion System for Faraday Connected MHD Generators," Paper No. 92 SM 520-7 EC, presented to the IEEE Power Engineering Society Summer Meeting, Seattle, WA, July 12-16, 1992.

[2]R. M. Johnson, M. K. Donnelly, "A Pulse-AmplitudeSynthesis-and-Control (PASC) Inversion System For Single-Source Diagonally Connected MHD Generators," Paper No. 93 WM 055-4 EC, presented to the IEEE Power Engineering Society Winter Meeting, Columbus $\mathrm{OH}$, Jan. 31-Feb. 5, 1993.

[3]B. K. Bose, "Evaluation of Modern Power Semiconductor Devices and Future Trends of Converters," IEEE Transactions on Industry Applications, Vol. 28, No. 2, pp. 403-413, March/April 1992.

[4]R. M. Johnson, K. Marcotte, M. K. Donnelly, "A Feasibility Demonstration Experimental Facility Simulation of a PASC Consolidation/Inversion System for Faraday Connected MHD Generators," Proceedings of the 28th
Symposium on Engineering Aspects of Magnetohydrodynamics (SEAM), Chicago, Il, June 28-30, 1990.

[5]R. M. Johnson, K. Marcotte, M. K. Donnelly, Computer Controlled MHD Power Consolidation and Pulse Generation System, Final Technical Progress Report to U.S. Department of Energy, Pittsburgh Energy Technology Center, Contract DE-AC22-87PC79680, Aug. 1990.

[6]B.A. Francis, and W.M. Wonham, "The Internal Model Principle of Control Theory," Automatica, vol. 12, pp.457-465, 1976.

[7]G.F. Franklin, and J.D. Powell, Feedback Control of Dynamic Systems, Reading, Massachusets: Addison-Wesley Publishing Co., 1987.

Matthew K. Donnelly (Member) was born in Phoenix, Arizona in 1960 . He received his B.S. from the University of Arizona and M.S. and $\mathrm{PhD}$ degrees from Montana State University in Electrical Engineering. $\mathrm{He}$ is currently employed by Battelle, Pacific Northwest Laboratories in Richland, Washington, and has published several papers on power system stability analysis and power converter technologies.

Roy M. Johnson (Member) is Professor of Electrical Engineering at Montana State University. He received his B.S. degree from the University of California at Berkeley and the $\mathrm{PhD}$ in Electrical Engineering from the U.S. Naval Postgraduate School, Monterey, California in 1969. Dr. Johnson has worked in the area of magnetohydrodynamic power generation since the early 1970 s and has published numerous papers on electrical plasma transients in MHD ducts and power consolidation and solid-state power conversion technology as applied to MHD advanced energy systems. 

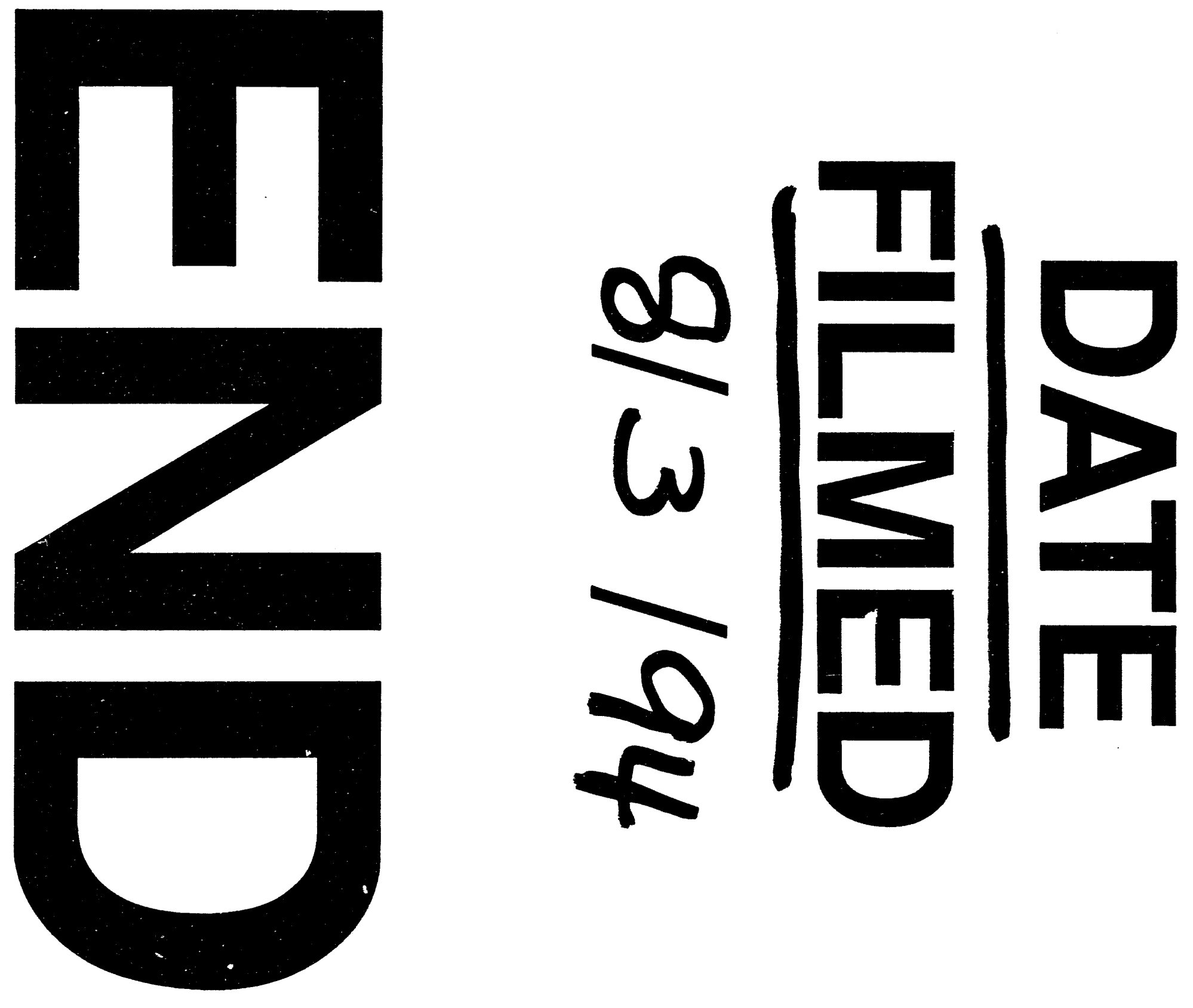
